\title{
Avaliação estrutural de pavimento de concreto continuamente armado de curta extensão para corredores de ônibus
}

\author{
Lucio Salles de Salles ${ }^{1}$, José Tadeu Balbo², Deividi da Silva Pereira ${ }^{3}$
}

\begin{abstract}
Resumo: Como uma solução alternativa de alta durabilidade para paradas e corredores de ônibus em áreas altamente urbanizadas, quatro seções experimentais de pavimento de concreto continuamente armado (PCCA) com diferentes taxas de armadura foram construídas no campus da Universidade de São Paulo. As seções possuem comprimento de apenas 50 metros, curtas em relação aos PCCA tradicionais, construídos o quanto o processo de concretagem permitir. Levantamentos de fissuras durante quatro anos indicaram que a curta extensão aliada à falta de ancoragem das seções fazem com que o padrão de fissuração seja muito diferente do PCCA tradicional; uma das seções ainda não apresentou fissuras na superfície. Para avaliar o desempenho estrutural do pavimento, dois ensaios não destrutivos foram realizados: primeiramente, testes de deflexão com falling weight deflectometer para determinar o valor da eficiência da transferência de carga entre fissuras e realizar a parametrização elástica por meio de retroanálise das seções de modo a obter o módulo de elasticidade do concreto (E) e o módulo de reação do subleito $(\mathrm{k})$; e, posteriormente, testes de carga dinâmicos para obter as tensões infligidas ao concreto por uma carga veicular conhecida. Resultados apontam um menor valor de E e k para pontos próximos a borda das seções e uma influência da frenagem nas tensões obtidas.
\end{abstract}

Palavras-chave: pavimento de concreto continuamente armado; fissuras; eficiência de transferência de carga; tensões dinâmicas

\begin{abstract}
As a proposed long-term pavement solution for bus stops and corridors in highly urbanized areas, four experimental short continuously reinforced concrete pavement (CRCP) sections with different percentages of longitudinal steel were built in São Paulo, Brazil. The pavement sections are only 50 meters long each, a short constructive length in comparison to traditional CRCP normally built as long as the concreting process allows. A four-year crack survey showed that the shorter length, and the consequential lack of anchorage, makes the short CRCP crack pattern to be unlike the traditional CRCP one; for instance, one of the pavement sections did not present any cracks yet. Two non-destructive tests were carried out to evaluate the short CRCP's structural performance: firstly, deflection tests using a falling weight deflectometer to evaluate layer elastic parameters through backcalculation, i. e., elastic modulus of concrete (E) and mudulos of subgrade reaction (k) and to determinate the load transfer efficiency across cracks; secondly, dynamic load tests to obtain the concrete stresses under a known truck axle load. The results show lower $\mathrm{E}$ and $\mathrm{k}$ for points near the slab edge and the braking influence on the stresses.
\end{abstract}

Keywords: Continuously Reinforced Concrete Pavement; Cracking; Load Transfer Efficiency; Dynamic Stresses.

\section{INTRODUÇÃO}

O grande número de pavimentos asfálticos em corredores de ônibus que apresentaram problemas como afundamento e ondulações, relacionados às elevadas cargas dos veículos e às altas temperaturas típicas do país, fez com que houvesse a substituição desses pavimentos por pavimentos de concreto simples (PCS). No entanto, patologias recorrentes como escalonamentos e esborcinamentos nas juntas induziram a fracassos com pouco tempo de vida útil dos PCS. Além disso, juntas defeituosas causam irregularidade no pavimento, reduzindo sua qualidade de rolamento, conforme comentam Liu et al. (2008).

Segundo Balbo (2009), dentre as seis principais causas de falhas em idades jovens dos PCS no Brasil, quatro são relacionadas às juntas construtivas de contração. Sejam por problemas no projeto ou por uma fraca prática construtiva, falhas nas juntas de contração e nas barras de transferência de carga são o indicador chave de um desempenho

\footnotetext{
${ }^{1}$ Lucio Salles de Salles, Departamento de Engenharia de Transportes, Escola Politécnica da Universidade de São Paulo. (lucio.salles@usp.br) 2 José Tadeu Balbo, Departamento de Engenharia de Transportes, Escola Politécnica da Universidade de São Paulo. (jotbalbo@usp.br)

${ }^{3}$ Deividi da Silva Pereira, Departamento de Engenharia de Transportes, Universidade Federal de Santa Maria, Santa Maria. (dsp@ufsm.br)

Manuscrito recebido em 19/08/2014 e aprovado para publicação em 01/06/2015.

Este artigo é parte de TRANSPORTES v. 23, n.2, 2015. ISSN: 2237-1346

(online). DOI: 10.14295/transportes.v23i2.835
}

anômalo dos PCS. Agora, se a maioria dos defeitos do PCS está nas juntas, por que não utilizar um pavimento de concreto sem juntas?

É nessa premissa que se insere a ideia do pavimento de concreto continuamente armado (PCCA): um pavimento de concreto sem juntas, uma placa contínua apenas com juntas construtivas ao final da jornada diária de trabalho.

Nos PCS, através da serragem das placas, é possível induzir/controlar as fissuras em uma área onde existem barras de transferência que serão responsáveis pela transferência de carga entre placas. Para o PCCA, não há diferença no comportamento do concreto, ele ainda fissura. A disparidade em relação aos PCS é a presença de uma alta taxa de armadura, posicionada pouco acima da linha neutra da placa, cuja finalidade precípua é manter as inevitáveis fissuras fortemente apertadas, de tal maneira que a sua existência seja imperceptível ao rolamento e que a transferência de carga pelo intertravamento dos agregados e pela armadura seja elevada. Não há, então, um controle e nem uma indução dessa fissuração, pois existe toda uma estrutura de aço projetada para manter a integridade estrutural e funcional da placa (Tayabji et al., 1995; Kohler e Roesler, 2004).

O sucesso deste tipo de estrutura é dado em função, basicamente, da sua durabilidade e pouca necessidade de manutenção. Para elucidar os principais atrativos do PCCA, trazem-se quatro diferentes estudos: 
1. Dossey e Hudson (1994), analisando pavimentos executados entre 1974 e 1987, calcularam que a primeira intervenção pesada de manutenção (recapeamento) nos PCCA ocorre em média aos 17 anos de serviço, mas que para a maioria das estruturas, o overlay só foi necessário a partir dos 20 anos de vida;

2. Tayabji et al. (1995) determinaram o International Roughness Index (IRI) - parâmetro que qualifica o rolamento do pavimento - para vários PCCA no Texas; os autores chegaram a valores entre $0,84 \mathrm{~m} / \mathrm{km}$ e $2,48 \mathrm{~m} / \mathrm{km}$, o que indica uma ótima qualidade de rolamento visto que a idade dos pavimentos estava entre 0,3 e 22 anos;

3. Gharaibeh et al. (1999), ao analisarem dados de diversos PCCA construídos entre 1977 e 1994, concluíram que, apesar de diferenças nos projetos, todos os pavimentos suportaram tráfego maior do que aquele para o qual foram projetados; ademais, todos os pavimentos avaliados duraram mais que sua vida de projeto;

4. Won (2011), também analisando PCCA no Texas, concluiu que mesmo pavimentos com 33 anos de exposição ao tráfego ainda possuíam as fissuras bastante fechadas na superfície e que, a despeito da idade do pavimento e da presença de defeitos, a eficiência da transferência de carga nas fissuras era sempre superior a $90 \%$.

Incentivado por anos de sucesso internacional comprovado, foi executado o primeiro trecho experimental de PCCA do Brasil no campus da Universidade de São Paulo (USP) em São Paulo no ano de 2010. São quatro seções de proposital curta extensão para simular o desempenho deste pavimento como uma parada de ônibus, situação crítica em infraestrutura viária urbana.

A possibilidade de fracasso na execução deste pavimento em temperaturas elevadas é muito mais alta do que em temperaturas baixas, típicas do clima temperado, como afirmam Schindler e McCullough (2002) e McCullough e Dossey (1999). Estudos nacionais também chamam atenção para os efeitos do gradiente térmico em placas de concreto (Pereira e Balbo, 2001; Rodolfo e Balbo, 2010).

Em Salles et al. (2012) e de maneira atualizada em Salles et al. (2013), foi apresentado e discutido o mapa de fissuração do PCCA curto; notou-se que a falta de ancoragem no final das seções aliada à curta extensão e à presença de uma base em camada asfáltica facilitaram a movimentação da massa de concreto nos primeiros momentos após a construção. Esse fator aliado ao poder de ligação oferecido pela armadura longitudinal esconde e atrasa o surgimento das fissuras na superfície.

Este artigo traz os resultados de dois testes não destrutivos com o objetivo de verificar a resposta estrutural do pavimento por meio da retroanálise de parâmetros elásticos e análise de tensões dinâmicas de forma a indicar se as diferenças no padrão de fissuração do PCCA curto implicam em seu desempenho.

\subsection{Pista experimental}

Primeiramente apresentada por Salles et al. (2012), as seções da pista experimental foram construídas entre julho e setembro de 2010, no período seco do inverno paulistano, com extensão de $50 \mathrm{~m}$ e largura de 5,05 m cada uma.
O tráfego diário é composto de, aproximadamente, 800 ônibus urbanos junto com algumas dúzias de caminhonetes médias e 1.500 veículos de passeio.

As seções são compostas por camadas idênticas de sub-base com macadame seco $(300 \mathrm{~mm})$, base de concreto asfáltico $(60 \mathrm{~mm})$ - relatórios de outros estudos apontam que bases asfálticas trabalham melhor do que granulares ou cimentadas (Gharaibeh et al., 1999; Johnston e Surdahl, 2006) - e de placas de concreto (240 mm); a única diferença entre as seções é a porcentagem de armadura longitudinal, distribuídas da seguinte forma: seção $1(0,6 \%)$; seção 2 $(0,7 \%)$; seção $3(0,4 \%)$; e seção $4(0,5 \%)$. A armadura transversal é composta de barras espaçadas $0,9 \mathrm{~m}$ entre si; todas as barras possuem diâmetro de $20 \mathrm{~mm}$ (aço CA-50). A resistência à tração na flexão de projeto do concreto foi fixada em 4,5 MPa (aos 28 dias).

Não há ancoragem no final das seções, dando liberdade ao deslocamento longitudinal de placas de concreto, como seria no caso de construções em paradas de ônibus de corredores urbanos (sem laje de transição ou ancoragem). Nota-se que, salvo a porcentagem de armadura longitudinal, as quatro seções possuem as mesmas características de projeto. A Figura 1 traz o mapa atualizado das fissuras.

\section{ESTUDO DE DEFLEXÕES}

Através de aplicações de FWD no meio das placas, entre as fissuras nas seções 2, 3 e 4 e a cada 10 m na seção 5 (pontos $\mathrm{P}$ na Figura 1) e aplicações tangenciais à fissura (Pontos F), foi possível traçar as bacias de deformação conforme mostram as Figura 2 e 3 . A carga aplicada pelo FWD foi de $60 \mathrm{kN}$ em função da necessidade de um melhor detalhamento das leituras de deflexões, fato que poderia ficar prejudicado com a aplicação de carregamentos inferiores que gerariam deflexões imperceptíveis em função da elevada rigidez de um pavimento de concreto com altas taxas de armadura.

Primeiramente, nota-se que a menor deflexão máxima ocorre na maior placa formada entre fissuras, a P2.2 no meio da seção 2. Isso pode ser explicado pela maior taxa de armadura da seção o que lhe confere uma maior rigidez. Já as maiores deflexões máximas - valores acima de 25x10$2 \mathrm{~mm}$ incidiram justamente nas bordas transversais das seções. A falta de ancoragem nestes pontos elucida o porquê deste fato; nestes locais a placa está mais livre para deslocar-se e não incomum é a sensação de um deslocamento vertical quando um veículo pesado acessa ou sai da via.

Apesar de sua maior taxa de armadura em relação às seções 3 e 4, a seção 1 apresenta o local (P1.1) com a maior deflexão máxima (38x10-2 mm) - bastante superior ao segundo colocado, o ponto P4.1 (seção 4) com 31,4x10-2 mm; isso ocorre porque há um afundamento no pavimento intertravado na junta com a pista experimental. Esse defeito já causou o escalonamento e a quebra do canto da placa. Também é interessante notar que os dois locais com os maiores deslocamentos são aqueles de acesso do veículo do pavimento intertravado para a pista experimental. Quanto ao formato, salvo a bacia P1.1, as bacias aparentam um contorno típico de pavimentos rígidos com uma diminuição de deflexões suave através dos leitores.

Na Figura 3, observa-se a inexistência de valores muito elevados da deflexão máxima, conforme esperado, a 

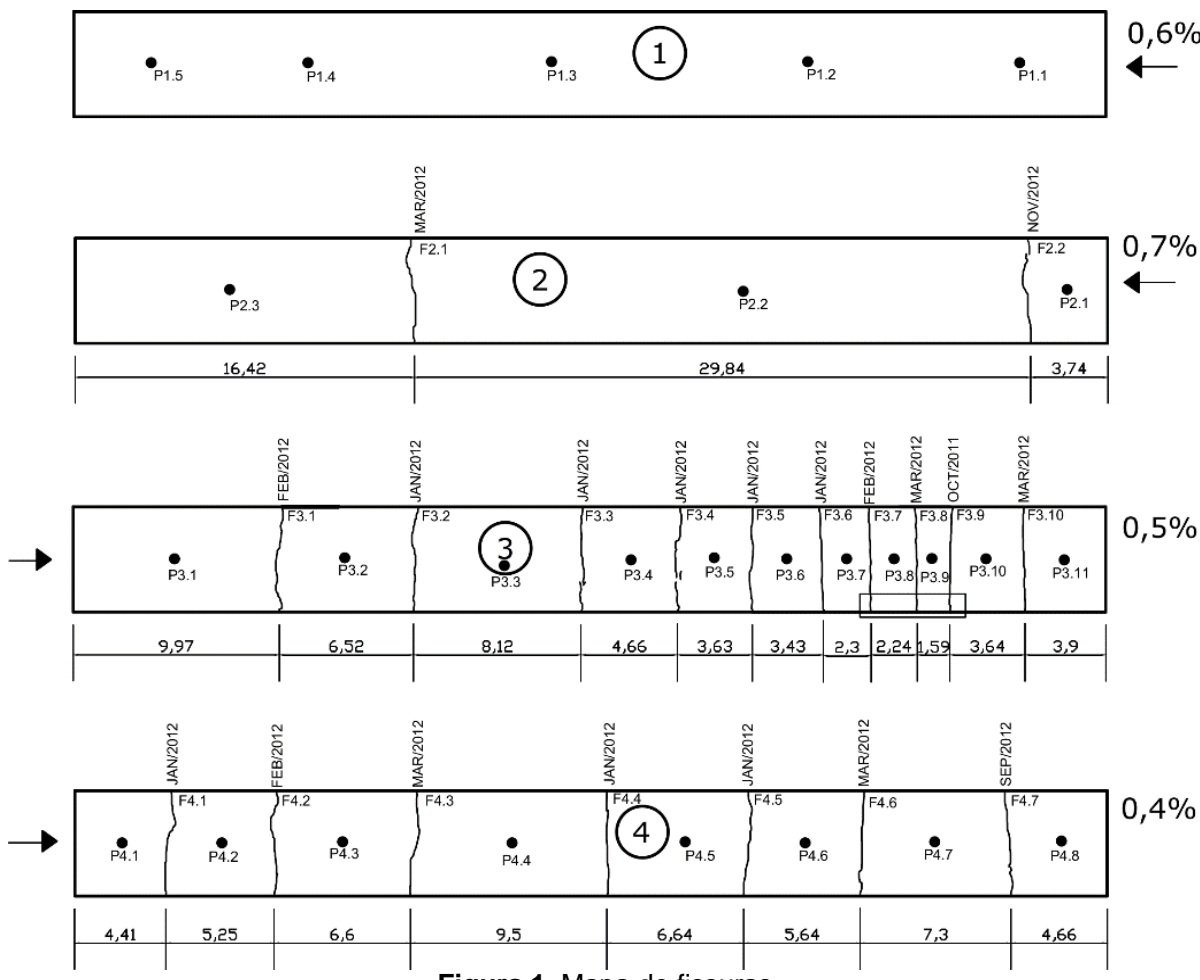

Figura 1. Mapa de fissuras

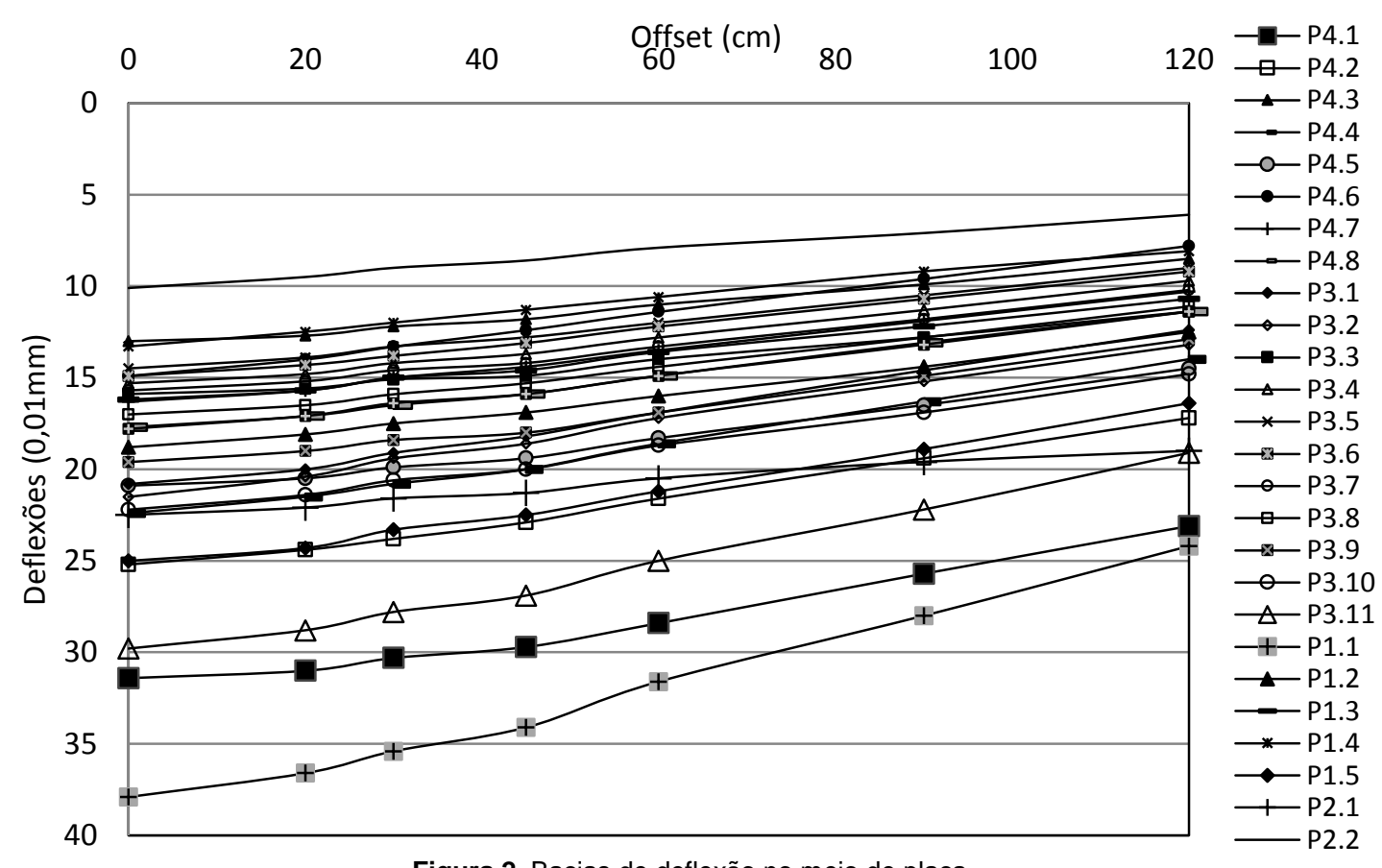

Figura 2. Bacias de deflexão no meio de placa

maior distância da fissura em relação à borda livre da placa atenua os deslocamentos. Porém, novamente, as duas maiores deflexões máximas são de pontos próximos à borda (F3.10 e F4.1). Não foi observado um aumento significativo de deflexões ao comparar-se as medidas tomadas na fissura e no meio da placa em locais próximos - em alguns casos as deflexões medidas na fissura foram até inferiores.

Colim et al. (2011) evidenciou, em um PCS, que juntas sem a barra de transferência de carga sofriam deslocamentos muito superiores àqueles apresentados por juntas com o dispositivo. Apesar das juntas (fissuras) do PCCA não possuírem barras de transferência de carga, a pequena abertura das fissuras somada à força de ligação da armadura longitudinal mantêm a placa íntegra, aumentando a rigidez e diminuindo as deflexões mesmo em uma descontinuidade física.

\subsection{Eficiência de transferência de carga (LTE) nas fissuras}

Para determinar a LTE das fissuras, a metodologia mais simples e mais corriqueiramente utilizada é aquela introduzida por Shahin (1985); nela, a porcentagem de transferência de carga é obtida por meio de testes com aplicação de carga adjacente à fissura. As deflexões são mensuradas 


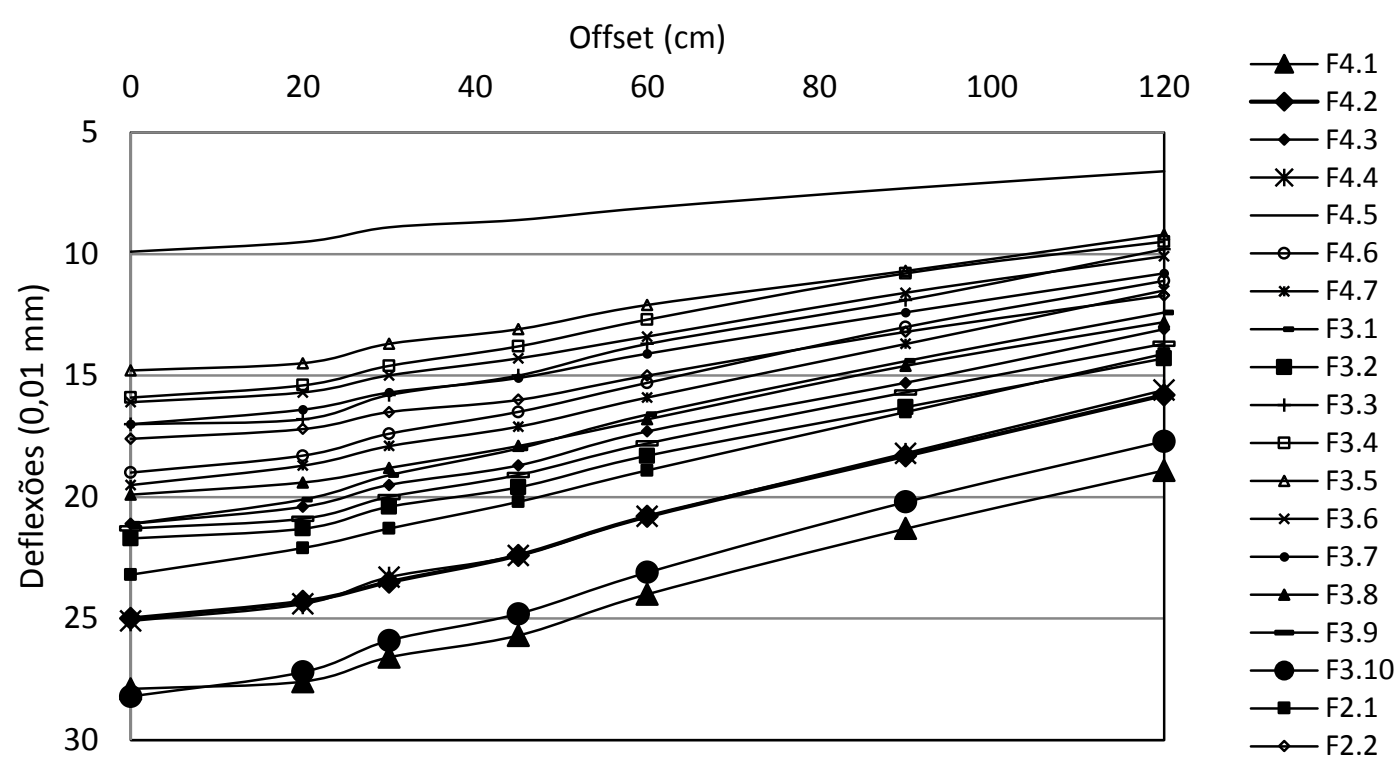

Figura 3. Bacias de deflexão na fissura

na placa sob a carga aplicada e na placa sem o carregamento à uma mesma distância da fissura, ou seja, são medidas de deflexão em simetria. O cálculo é realizado através da Equação 1.

$$
L T E=\frac{\delta_{2}}{\delta_{1}} \times 100[\%]
$$

Onde:

$\delta_{1}=$ Deflexão na placa carregada;

$\delta_{2}=$ Deflexão na placa sem carregamento.

Os resultados mostraram que todas as fissuras apresentaram um valor de LTE maior que $90 \%$ (média de $92,7 \%$ ). Não foi possível observar qualquer influência da porcentagem de armadura longitudinal na LTE; esperava-se que as fissuras da seção 2 apresentassem os maiores valores, entretanto as médias das três seções foram bastante similares. A fissura com o menor valor de LTE (90,52\%) é a F3.1, posicionada no fim da seção 3; todavia, estranhamente, a fissura com o maior valor de LTE (F4.1 - 95,51\%) também encontra-se no limite de uma seção (no caso, seção 4). As deflexões nesta posição foram elevadas conforme visto nas Figuras 2 e 3, entretanto, o maior ou menor deslocamento não parece influenciar no comportamento de transferência de carga na fissura.

Conforme alguns pesquisadores confirmam, a influência do espaçamento também não foi observada nos valores do LTE. No presente estudo, igualmente, nenhuma tendência foi notada, ou seja, espaçamentos muito grandes como aqueles apresentados pela seção 2 não são prejudiciais à transferência de carga. Outro aspecto é que ao simular uma fissura nos pontos de aplicação no meio da placa e calcular a LTE, os valores de transferência de carga resultam muito similares àqueles apresentados na fissura. Isso significa que, em relação à transferência de carga, a fissura do PCCA de curta extensão comporta- se como um pavimento contínuo.

\subsection{Parametrização por meio de retroanálise}

O processo de retroanálise, através da equiparação de bacias de campo (reais) com bacias teóricas, permite a de- terminação dos valores do módulo de elasticidade do concreto $(\mathrm{E})$ e do módulo de reação do subleito (k). As deflexões teóricas foram obtidas por meio do software EverFE (Davids, 2004); embora que o programa tenha sido desenvolvido para análises em PCS, é possível simular outras estruturas conhecendo a geometria delas. Portanto, no caso do PCCA, as fissuras foram consideradas como juntas sem barras de transferência.

Deve-se notar que a armadura longitudinal não tem um papel estrutural no PCCA; ela somente mantém as fissuras fortemente unidas, conforme comentam Zollinger e Barenberg (1990). Como consequência dessa função, a transferência de carga nas fissuras ocorre exclusivamente pelo intertravamento de agregados, o que é proporcional à rigidez da fissura/junta. Os valores de rigidez da fissura foram baseados em relações com a abertura da fissura evidenciados por Jeong e Zollinger (2001).

Os valores preliminares de $\mathrm{E}$ e $\mathrm{k}$ foram estimados de acordo com os estudos de Hall (1991) e Crovetti (1997). O processo de equiparação das bacias foi conduzido com quantas simulações fossem necessárias para atingir um mínimo erro quadrático, o qual foi calculado pela Equação 2.

$$
\text { Erroquadrático }=\sum\left(d_{i}^{\text {Real }}-d_{i}^{\text {Teórica }}\right)^{2}
$$

Todas as aplicações de FWD entre as fissuras e na seção 1 (pontos $\mathrm{P}$ ) foram consideradas para retroanálise. A Tabela 1 traz os valores de E e k retroanalisados. Durante o processo, foi observado que a equiparação de bacias próximas a borda resultaram em deflexões teóricas muito altas e, por consequência, valores baixos de E e k. Isso é motivado novamente pela falta de ancoragem que permite deslocamentos verticais livres, o que por sua vez, faz com que o concreto sofra mais fortemente os impactos de veículos causando uma deterioração precoce. Como esperado, o ponto P1.1 apresentou os menores valores de E e k em função da severa deterioração do concreto causada pelos defeitos no pavimento intertravado próximo à junta. A seção 2 apresentou os maiores valores de $\mathrm{E}$ e $\mathrm{k}$, inclusive quando a aplicação da carga foi próxima da borda, o que é explicado pela alta taxa de armadura da seção que aumenta sua rigidez. 
Tabela 1. Parâmetros retroanalizados para cada aplicação de FWD

\begin{tabular}{|c|c|c|c|c|c|c|c|c|c|c|c|c|c|c|}
\hline & Seção 1 & & & & & Seção & & & & & & & \multirow{2}{*}{\multicolumn{2}{|c|}{ P4.8 }} \\
\hline Ponto & P1.1 & $P 1.2$ & P1.3 & $P 1.4$ & P1.5 & $P 4.1$ & P4.2 & $P 4.3$ & P4.4 & P4.5 & P4.6 & P4.7 & & \\
\hline E (MPa) & 12.000 & 35.000 & 34.000 & 38.000 & 28000 & 25.000 & 27.000 & 45.000 & 25.000 & 30.000 & 28.000 & 32.000 & \multicolumn{2}{|c|}{30.000} \\
\hline $\mathrm{k}(\mathrm{MPa} / \mathrm{m})$ & 53 & 95 & 120 & 160 & 70 & 53 & 70 & 145 & 85 & 85 & 155 & 125 & \multicolumn{2}{|l|}{115} \\
\hline $\begin{array}{l}\text { Erro Qua- } \\
\text { drático }\end{array}$ & 17,18 & 0,53 & 0,43 & 0,39 & 1,12 & 0,96 & 0,29 & 0,22 & 0,21 & 0,56 & 0,24 & 0,29 & \multicolumn{2}{|l|}{0,27} \\
\hline & \multicolumn{3}{|c|}{ Seção 2} & \multicolumn{11}{|c|}{ Seção 3} \\
\hline Ponto & $P 2.1$ & $P 2.2$ & $P 2.4$ & $P 3.1$ & $P 3.2$ & $P 3.3$ & P3.4 & P3.5 & P3.6 & $P 3.7$ & P3.8 & P3.9 & P3.10 & $P 3.11$ \\
\hline $\mathrm{E}(\mathrm{MPa})$ & 30.000 & 60.000 & 35.000 & 25.000 & 25.000 & 30.000 & 35.000 & 42.000 & 38.000 & 42.000 & 42.000 & 28.000 & 28.000 & 25.000 \\
\hline $\mathrm{k}(\mathrm{MPa} / \mathrm{m})$ & 80 & 200 & 105 & 95 & 95 & 120 & 130 & 135 & 135 & 115 & 105 & 100 & 85 & 60 \\
\hline $\begin{array}{l}\text { Erro Qua- } \\
\text { drático }\end{array}$ & 17,23 & 0,16 & 0,11 & 0,37 & 0,90 & 3,60 & 0,22 & 0,23 & 0,20 & 0,44 & 0,27 & 0,44 & 0,48 & 0,82 \\
\hline
\end{tabular}

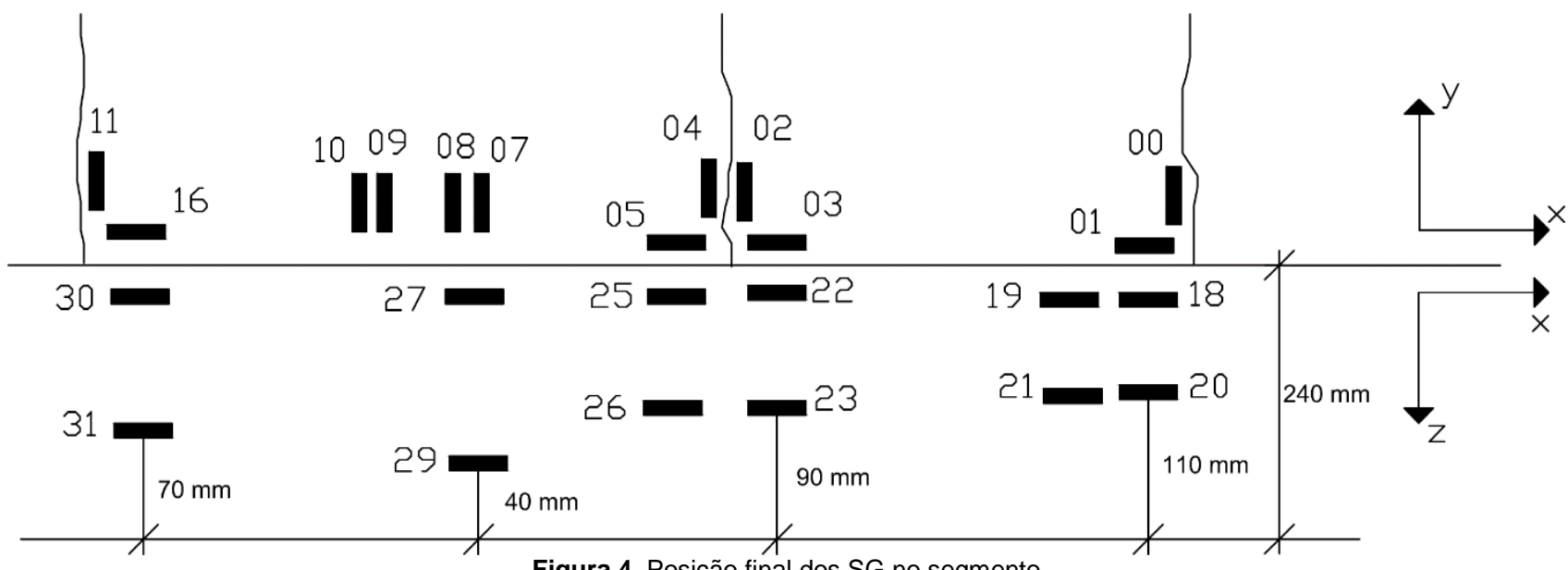

Figura 4. Posição final dos SG no segmento

\section{PROVAS DE CARGA DINÂMICAS (PCD)}

O segmento para instrumentação (retângulo na Figura 1) encontra-se na seção 3 e foi selecionado com base no maior número de fissuras com o menor espaçamento. Em um dia seco de fevereiro de 2013 (aproximadamente $20^{\circ} \mathrm{C}$ de temperatura) foi realizada a remoção da sarjeta e a instrumentação da pista. Uma divisória de isopor foi encontrada entre a placa e a sarjeta o que tornou a primeira bastante suave e regular, facilitando o posicionamento dos strain gauges (SG). Entretanto, essa divisória possuía apenas $200 \mathrm{~mm}$ contra os $240 \mathrm{~mm}$ da placa, fazendo com que se formasse um "pé de concreto" nos últimos centímetros, impossibilitando a colagem dos SG no fundo efetivo da placa. Na Figura 4 é exibida a posição de cada SG.

O caminhão-teste apresentara configuração de eixo simples de rodas simples (ESRS) dianteiro e eixo simples de rodas duplas (ESRD) traseiro com uma carga por eixo de 3.910 e $12.580 \mathrm{~kg}$, respectivamente. Como referência da distância dos eixos e dos SG, foi pintada, na borda da placa, uma faixa à exatos $30 \mathrm{~cm}$ da borda externa. Foram realizadas 15 PCD com velocidade constante de $10 \mathrm{~km} / \mathrm{h}$, três com alta velocidade $(40 \mathrm{~km} / \mathrm{h})$ e duas com frenagem no meio do segmento. A temperatura do teste foi controlada por um termômetro posicionado na superfície da pista e atingiu uma média de $20,9^{\circ} \mathrm{C}$ durante o ensaio.

\subsection{Análise de tensões}

Primeiramente, para visualizar e compreender os sinais de passagem dos dois eixos pelo trecho-teste, na Figura 5 é apresentado o gráfico deformação versus tempo. Nele estão explícitas as deformações causadas pelos eixos do caminhão durante o tempo de ensaio da prova de carga dinâmica 20 (PCD20) no SG 16. A PCD20 foi realizada com uma velocidade média de $10 \mathrm{~km} / \mathrm{h}$, com frenagem na posição dos SG 08 e 07.

A escolha do SG 16 deu-se em função desse instrumento coletar as deformações resultantes da passagem dos dois eixos nas duas provas de carga; como o caminhão parou na posição dos $\mathrm{SG} 8$ e 7, os únicos $\mathrm{SG}$ capazes de medir deformações são os anteriores a eles.

Para verificar o efeito da passagem de uma carga em um gráfico deformação versus tempo é necessário visualizar as alterações na deformação. Deve-se ter cuidado na análise dos dados, pois muitas vezes fatores externos podem modificar a leitura de deformação do instrumento. No caso do SG 16 fica clara a passagem dos eixos do veículo na seguinte sequência: aos 7,2 $\mathrm{s}$ do ensaio o ESRS solicitou a placa no exato local do SG 16 causando o primeiro pico decrescente; um segundo depois foi a vez do ESRD solicitar a placa originando o segundo pico; o caminhão parou no segmento, aos 9,2 s onde permaneceu por aproximadamente $8 \mathrm{~s}$; aos 17,2 s, acelerando em marcha à ré, o ERSD passa novamente pelo SG 16 criando o terceiro pico e é seguido $3 \mathrm{~s}$ após pelo ESRS finalizando a passagem completa com o quarto pico. Com as informações do tempo de passagem de ambos os eixos e o conhecimento da configuração do caminhão, também foi possível determinar a velocidade de operação do veículo em cada etapa do ensaio.

As tensões de tração ou compressão na flexão geradas $(\sigma)$ na direção específica do SG são o produto da multiplicação entre o diferencial de deformação entre os estados de relaxação e tensional máximos $(\Delta \varepsilon)$ e módulo de elasticidade do material. Se o valor do $\Delta \varepsilon$ for positivo, significa que aquele ponto específico da placa sofreu tração na flexão; caso contrário, se $\Delta \varepsilon$ for negativo, o carregamento induziu compressão na flexão no determinado local. A Figura 


\section{DLT20 - SG16}

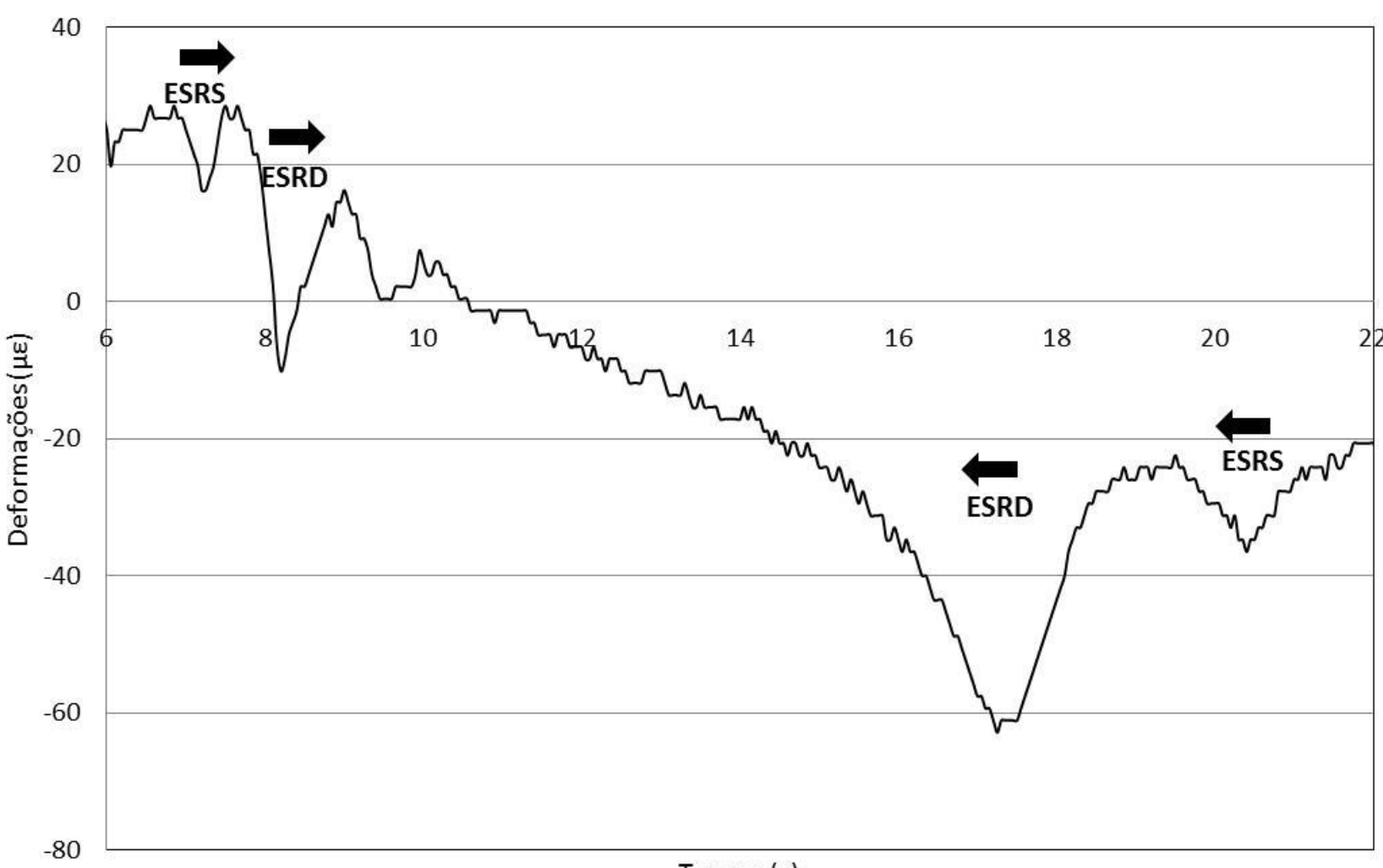

Tempo(s)

Figura 5. Visualização das passagens dos eixos na PCD20

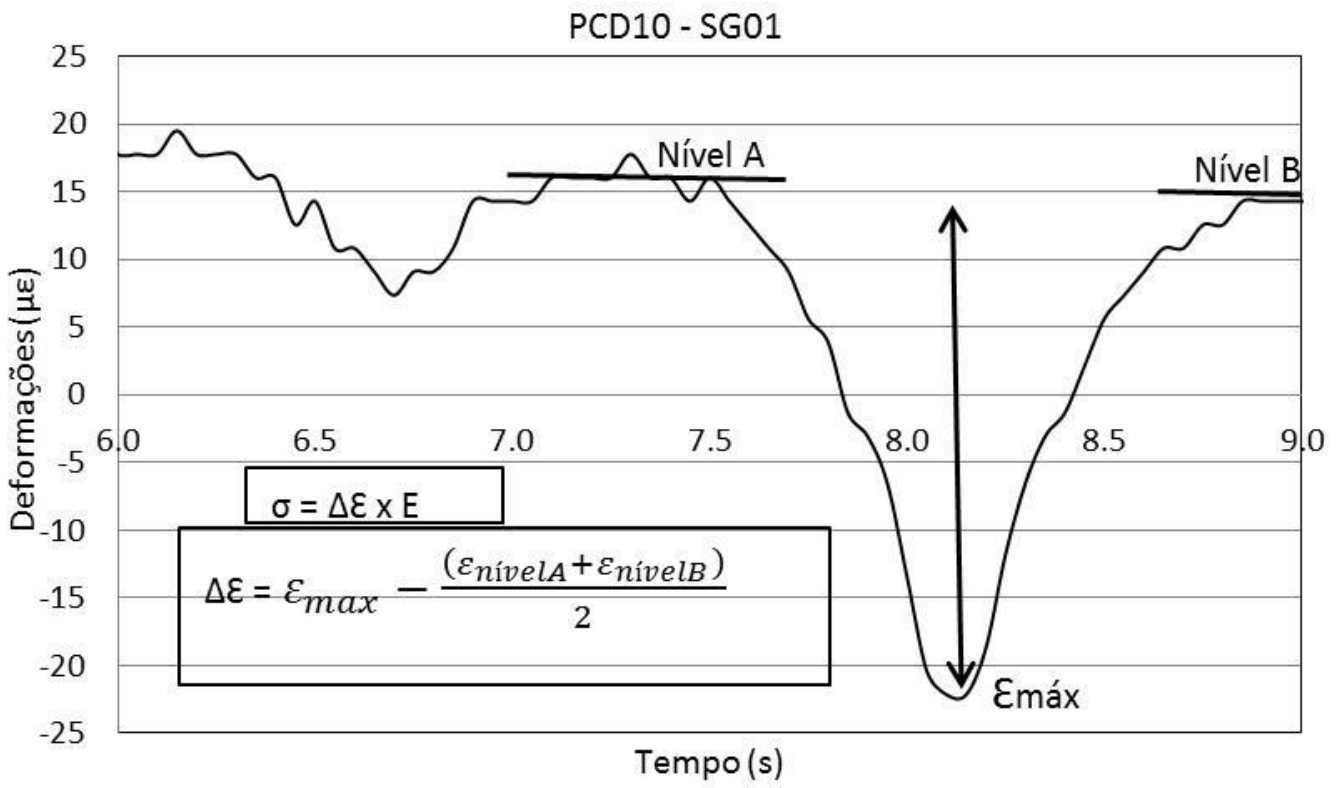

Figura 6. Método de cálculo de tensões na PCD10

6 exemplifica o método de cálculo juntamente com a equação utilizada. O módulo de elasticidade (E) utilizado foi aquele retroanalisado para os pontos específicos onde estão fixados os SG, presentes na Tabela 1.

De maneira previsível, as tensões geradas pelo ESRS foram inferiores àquelas obtidas com o ESRD em função da maior carga do segundo. A teoria de que os instrumentos posicionados na superfície - ou próximos ao topo - e no fundo da placa iriam medir tensões de compressão (negati- vas) e de tração (positivas), respectivamente, foi comprovada parcialmente. Todos os SG dispostos longitudinalmente responderam de acordo com a teoria; entretanto, os instrumentos fixados na superfície em posição transversal à placa (SG 07, 08, 09, 10 e 11) mediram picos de deformação positivos, o que gerou tensões de tração. Isso ocorre, supostamente, em razão do carregamento solicitar a carga não exatamente na borda. O deslocamento da passagem da carga pode criar, transversalmente, uma configuração de tensões (Figura 7) na qual abaixo da carga surgem tensões 

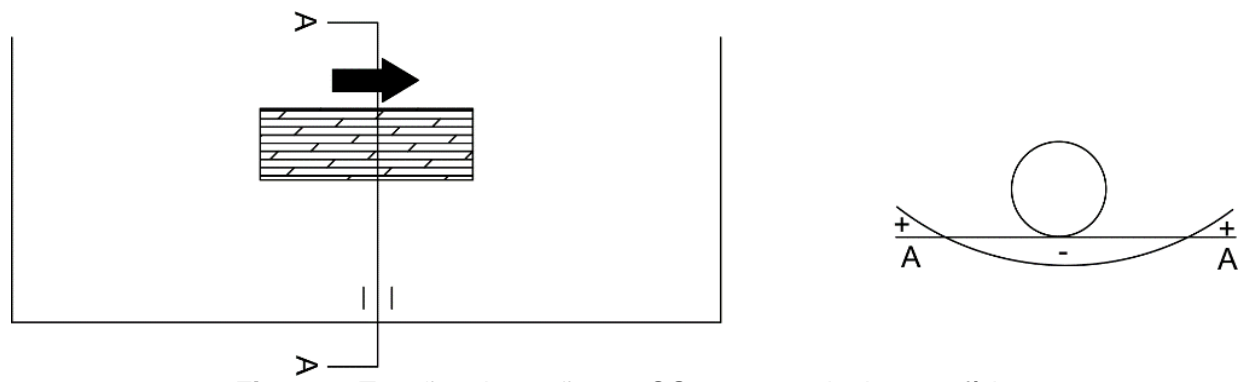

Figura 7. Tensões de tração nos SG transversais de superfície

Distância da borda $(\mathrm{cm})$

$\begin{array}{llllll}0 & 20 & 40 & 60 & 80 & 100\end{array}$

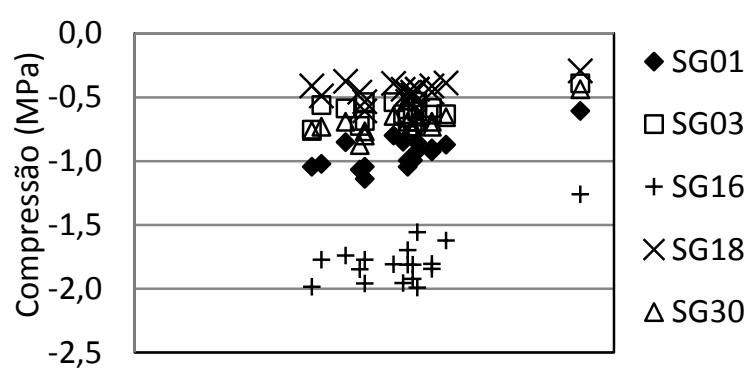

(a)

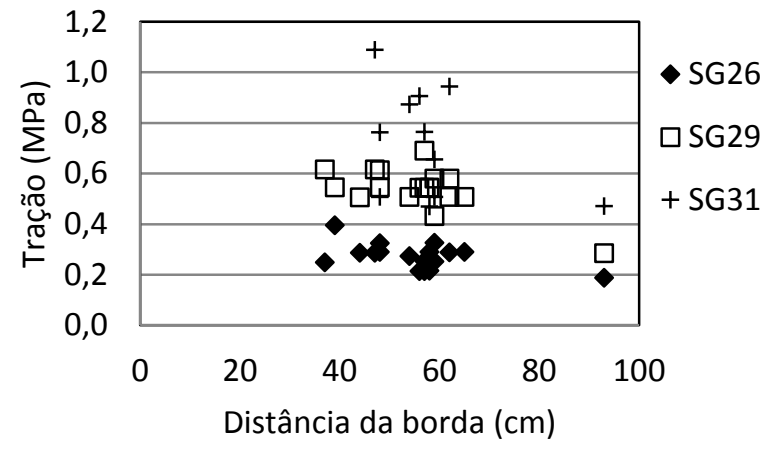

(c)
Velocidade média $(\mathrm{km} / \mathrm{h})$

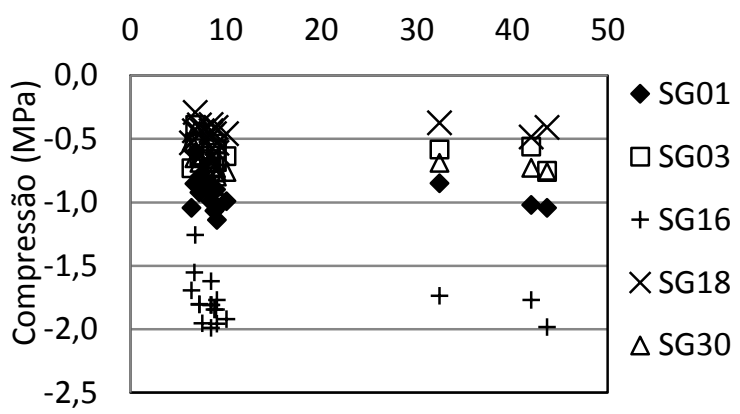

(b)

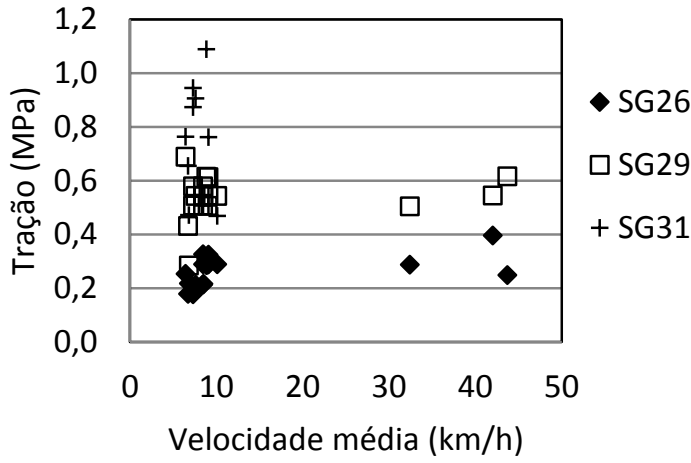

(d)

Figura 8. Tensões de compressão versus distância da borda (a) e velocidade média (b); tensões de tração versus distância da borda (c) e velocidade média $(\mathrm{d})$

de compressão e nas extremidades da placa tensões de tração.

A Figura 8 traz as tensões de compressão e de tração na flexão geradas pelo ESRD em relação à velocidade média do caminhão e a distância do ESRS da borda da placa. O grupo de gráficos mostra resultados coerentes para a maioria dos SG longitudinais (cabe salientar que alguns instrumentos não funcionaram, o que é compreensível para um experimento tão prático). Ao observar a distância de passagem do caminhão em relação aos instrumentos, é intuitivo que quanto mais distante for a solicitação menor será a deformação medida. Isso é facilmente percebível na PCD01 que passou à uma distância de $93 \mathrm{~cm}$ da borda - quase o dobro da média das outras PCD - criando os menores valores de tensões para todos os SG.

Quanto à velocidade do caminhão, nenhuma variação significativa de tensões foi observada no aumento da velocidade de 10 para $40 \mathrm{~km} / \mathrm{h}$, em uma contradição aberta com outros estudos que constataram que cargas com velocidades baixas ou quase estáticas são mais destrutivas (causam maiores tensões) para o pavimento (Kim et al., 2002; Xiao et al., 2011; Zhang et al., 2007; Silva e Motta, 1999)

\subsection{Efeito da frenagem}

Na Figura 9 é encontrada uma compilação dos resultados de tensão medidos pelas PCD com velocidade constante de $10 \mathrm{~km} / \mathrm{h}$ (PCD01 a 15), PCD com frenagem em operação normal (PCD19 e 20) e com frenagem em marcha à ré (PCD20). Infelizmente os dados de retorno (em marcha à ré) da PCD19 não foram observáveis no gráfico.

As tensões originadas pelo ESRS sofreram aumento de $23 \%$ (compressão) e $65 \%$ (tração) quando o veículo estava freando. Inversamente, as tensões causadas pelo ESRD foram 18\% (compressão) e 63\% (tração) mais elevadas quando o veículo estava em velocidade constante sem frenagem. Essa disparidade ocorre porque a frenagem no eixo dianteiro induz a um deslocamento do centro de massa do veículo causando um incremento de carga na parte dianteira. Essa ideia também explica o fato de que as tensões do ESRD são menores nas PCD com frenagem quando comparadas com PCD com velocidade constante; no primeiro caso, o ESRD estaria momentaneamente menos carregado.

Relativo ao processo em marcha à ré na PCD20, os valores foram expressivamente superiores aos das PCD 


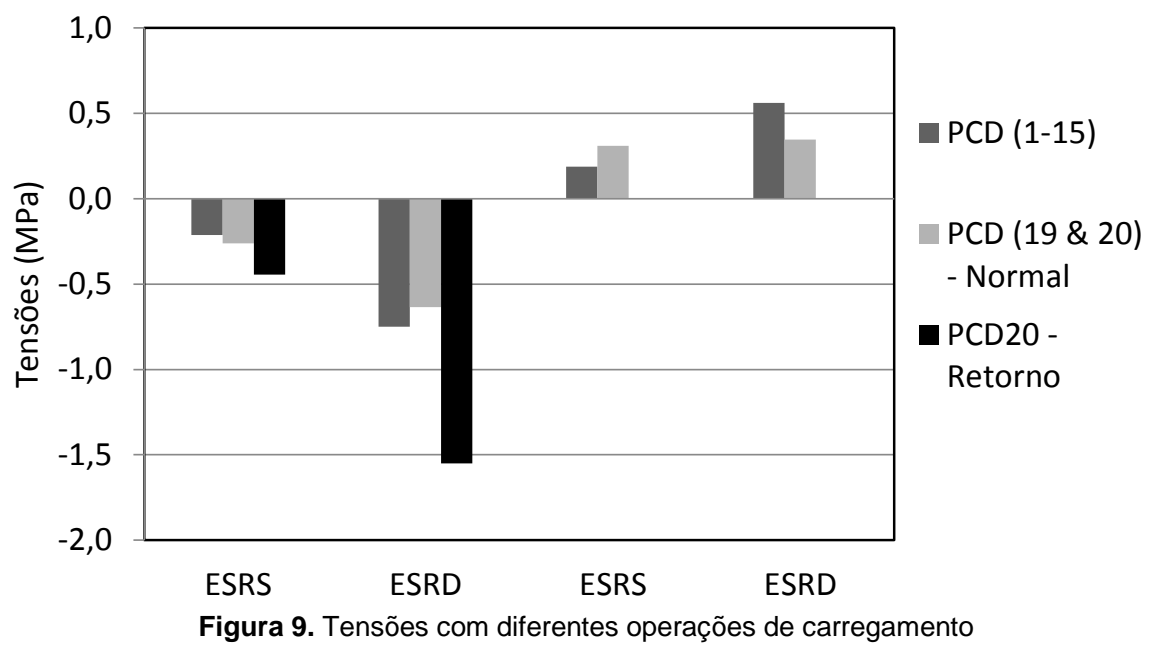

com velocidade constante; a operação de retorno foi realizada com um velocidade média de aproximadamente 4 $\mathrm{km} / \mathrm{h}$. Silva e Motta (1999) em pavimento experimental de whitetopping observaram as maiores deformações para essa mesma velocidade. Portanto, agora de acordo com estudos anteriores, as operações menos velozes causam maiores deformações na placa. Supostamente, somente velocidades inferiores a $10 \mathrm{~km} / \mathrm{h}$, ou seja, velocidades muito baixas são mais danosas à estrutura e velocidades acima de $10 \mathrm{~km} / \mathrm{h}$ são iguais em efeito.

\subsection{Análise de topo e fundo}

De posse dos valores de tensão de tração e compressão e com o conhecimento exato da posição destes SG foi possível traçar o diagrama de tensões atuantes na placa. Deve-se lembrar que, devido a um "pé" de concreto encontrado nos últimos centímetros da placa, os SG 26 e 31 foram fixados, respectivamente, a 90 e $70 \mathrm{~mm}$ do fundo da placa. Portanto o valor de tensão calculado não é propriamente do fundo; isso também ocorre com o topo, embora em menor escala - os instrumentos estão posicionados a $10 \mathrm{~mm}$ da superfície.

Através da extensão da linha de tensões do diagrama pôde-se estimar os valores das tensões máximas de fundo e de topo, além, claro, da determinação da posição da linha neutra (LN) na placa. Nota-se que a LN é inferida com relação ao fundo da placa. Estes dados encontram-se na Tabela 2 .

Pelos resultados, fica claro o incremento de tensões pela extrapolação do diagrama de tensões até o fundo "real" da placa. Vários instrumentos indicaram tensões acima de 1,0 MPa com o máximo valor superando os 3,0 MPa que, embora ainda abaixo da resistência de projeto do concreto (4,5 $\mathrm{MPa})$, sugere cuidado. A alta rigidez da placa retroanalisada na posição dos SG 31 e $30(42.000 \mathrm{MPa})$ pode explicar esses valores elevados; note que no caso dos SG 25 e 26 (rigidez de $28.000 \mathrm{MPa}$ ) nenhum valor ultrapassou 1,0 MPa.

A média da posição da $\mathrm{LN}$ foi de $155 \mathrm{~mm}$ a partir do fundo da placa. Em uma situação ideal, é esperado que a LN estivesse posicionada exatamente a meia altura da placa; porém, falhas na densificação, cura e mistura do concreto podem deslocar a LN. Para o PCCA de curta extensão o deslocamento encontrado foi de positivos $35 \mathrm{~mm}$ (acima da meia altura). Apesar dos fatores descritos acima, é intuitivo pensar que a armadura longitudinal (projetada $20 \mathrm{~mm}$ acima da meia altura) é que esteja deslocando a LN.

Tabela 2. Tensões no topo e fundo da placa e posição da linha neutra (LN)

\begin{tabular}{|c|c|c|c|c|c|c|c|c|c|c|}
\hline \multicolumn{7}{|c|}{$S G 30-31$} & \multicolumn{4}{|c|}{$S G 25-26$} \\
\hline \multirow[b]{2}{*}{$P C D$} & \multicolumn{3}{|l|}{ ESRS } & \multicolumn{3}{|l|}{ ESRD } & \multicolumn{4}{|c|}{$E S R D$} \\
\hline & $\begin{array}{l}\text { TOPO } \\
(\mathrm{MPa})\end{array}$ & $\begin{array}{l}\text { FUNDO } \\
(\mathrm{MPa})\end{array}$ & $\begin{array}{l}L N \\
(\mathrm{~mm})\end{array}$ & $\begin{array}{l}\text { TOPO } \\
(\mathrm{MPa})\end{array}$ & $\begin{array}{l}\text { FUNDO } \\
(M P a)\end{array}$ & $\begin{array}{l}L N \\
(\mathrm{~mm})\end{array}$ & $D L T$ & $\begin{array}{l}\text { TOPO } \\
(\mathrm{MPa})\end{array}$ & $\begin{array}{l}\text { FUNDO } \\
(M P a)\end{array}$ & $\begin{array}{l}L N \\
(\mathrm{~mm})\end{array}$ \\
\hline PCD01 & NA & NA & NA & $-0,494$ & 0,871 & 153,2 & DLT01 & $-0,324$ & 0,495 & 145,1 \\
\hline PCD02 & NA & NA & NA & $-0,735$ & 1,229 & 150,2 & DLT03 & $-0,371$ & 0,661 & 153,7 \\
\hline PCD03 & NA & NA & NA & $-0,739$ & 1,538 & 162,1 & DLT04 & $-0,600$ & 0,703 & 129,4 \\
\hline PCD04 & $-0,170$ & 0,435 & 170,4 & $-0,868$ & 1,638 & 156,8 & DLT06 & $-0,677$ & 0,814 & 131,0 \\
\hline PCD05 & NA & NA & NA & $-0,793$ & 1,661 & 162,4 & DLT08 & $-0,596$ & 0,705 & 130,0 \\
\hline PCD06 & NA & NA & NA & $-0,525$ & 1,328 & 164,8 & DLT09 & $-0,448$ & 0,733 & 148,9 \\
\hline PCD07 & NA & NA & NA & $-0,766$ & 1,033 & 137,8 & DLT10 & $-0,791$ & 0,995 & 133,7 \\
\hline PCD08 & NA & NA & NA & $-0,902$ & 3,196 & 187,2 & DLT11 & $-0,556$ & 0,796 & 141,3 \\
\hline PCD09 & $-0,224$ & 0,811 & 188,0 & $-0,789$ & 2,433 & 181,2 & DLT13 & $-0,563$ & 0,802 & 141,0 \\
\hline PCD10 & NA & NA & NA & $-0,845$ & 1,065 & 133,8 & DLT14 & $-0,561$ & 0,800 & 141,1 \\
\hline PCD12 & NA & NA & NA & $-0,997$ & 1,949 & 158,8 & DLT15 & $-0,517$ & 0,652 & 133,9 \\
\hline PCD13 & NA & NA & NA & $-0,897$ & 1,446 & 148,2 & DLT16 & $-0,447$ & 0,729 & 148,8 \\
\hline PCD14 & $-0,285$ & 0,422 & 143,5 & $-0,839$ & 1,006 & 130,9 & DLT17 & $-0,607$ & 0,998 & 149,2 \\
\hline PCD19 & $-0,249$ & 0,460 & 155,8 & NA & NA & NA & DLT18 & $-0,562$ & 0,737 & 136,2 \\
\hline PCD20 & $-0,136$ & 0,569 & 193,6 & $-0,507$ & 1,182 & 167,9 & & & & \\
\hline
\end{tabular}




\section{CONSIDERAÇÕES FINAIS}

Através de dois testes de avaliação estrutural não destrutivos, pode-se chegar as seguintes conclusões:

- Como as seções não possuem um sistema de ancoragem, a placa fica com as bordas livres, causando um maior deslocamento vertical. Em função disto, o módulo de elasticidade do concreto (E) e o módulo de reação do subleito $(\mathrm{k})$ retroanalisados resultaram em valores inferiores. Para o caso particular da borda da seção 1, os problemas apresentados pelo pavimento intertravado, próximo à pista experimental, estão causando uma forte deterioração do concreto, fato comprovado a partir do baixíssimo E retroanalisado:

- O LTE nas fissuras mostrou um comportamento adequado das seções. Todas as fissuras apresentaram um valor maior que $90 \%$. Pontos com maiores deflexões não implicaram em fissuras com baixas transferências de carga;

- As bacias obtidas tangentes às fissuras redundaram em resultados similares àquelas de meio de placa; as maiores deflexões estavam posicionadas nas bordas das placas. Ao simular uma fissura nas aplicações de meio de placa, notou-se que o valor da LTE foi muito parecido com o das fissuras reais. Esses resultados comprovam que o PCCA comporta-se como uma estrutura contínua;

- A maior porcentagem de armadura longitudinal da seção 2 aumenta a rigidez da placa, o que foi confirmado pelos elevados valores de E e k da seção. A armadura também confere maior rigidez à parte superior da placa, deslocando, de modo ascendente, a linha neutra em $35 \mathrm{~mm}$, conforme visto na análise tensões de topo e fundo da seção 3;

- A análise comparativa da frenagem mostrou que devido ao deslocamento do centro de massa do veículo durante essa operação, há um aumento nas tensões geradas pelo ESRS e uma diminuição nas originadas pelo ESRD. Velocidades de operação menores que $10 \mathrm{~km} / \mathrm{h}$ causam maiores tensões, enquanto que velocidades acima de $10 \mathrm{~km} / \mathrm{h}$ são iguais em efeito.

Por fim, apesar de todas as diferenças notadas na fissuração do PCCA de curta extensão, os dois estudos não destrutivos mostraram que o pavimento possui um comportamento estrutural adequado. As tensões de tração na flexão impostas pelo carregamento estão abaixo da resistência do concreto e a transferência de carga entre fissuras é elevada. Entretanto, deve-se considerar que um sistema de ancoragem ou até mesmo uma junta reforçada com o pavimento seguinte sejam necessários à proteção da placa contra deslocamentos verticais exagerados, fato que poderia levar à deterioração precoce do concreto.

\section{AGRADECIMENTOS}

Os autores são gratos à Fundação de Apoio à Pesquisa do Estado de São Paulo (FAPESP) pelo suporte fornecido ao estudo por meio do processo \# 98/11629-5 e a CAPES (Ministério de Educação) pela bolsa concedida ao primeiro autor. Agradecimentos também são direcionados à empresa Copavel pelo auxílio voluntário na execução do ensaio com o FWD.

\section{REFERÊNCIAS}

Balbo, J. T. (2009). Pavimentos de concreto. Oficina de Textos, São Paulo.

Balbo, J. T.; Massola, A.; Pereira, D. (2012). Structural aspects of the experimental CRCP in São Paulo. Proceedings of the 10th International Conference on Concrete Pavements, International Society for Concrete Pavements, Quebec.

Colim, G. M.; Balbo J. T.; Khazanovich, L. (2011) Effects of temperature changes on load transfer in plain concrete pavement joints. Ibracon Structures and Materials Journal, v. 4, p. 405437.

Crovetti, J. A. (1997) Design and evaluation of jointed concrete pavement systems incorporating open-graded permeable bases. $\mathrm{Ph}$. D. Dissertation, University of Illinois.

Davids, W. (2004). EverFE: Software for the 3D Finite Element Analysis of Jointed Plain Concrete Pavements. (Available at: www.civil.umaine.edu/everfe/).

Dossey, T.; Hudson, W. R. (1994). Distress as function of age in continuously reinforced concrete pavements: models developed for Texas pavement management information system. Transportation Research Record, v. 1455, p. 159 - 165.

Gharaibeh, N. G.; Darter, M. I.; Heckel, L. B. (1999). Field performance of continuously reinforced concrete pavement in Illinois. Transportation Research Record, v. 1684, p. 44 - 50. DOI: $10.3141 / 1684-06$

Hall, K. T. (1991) Performance, evaluation and rehabilitation of asphalt-overlaid concrete pavements. Ph.D. Dissertation. University of Illinois.

Jeong, J. H.; ZollingeR, D. G. (2001) Characterization of stiffness parameters in design of continuously reinforced and jointed pavements. Transportation Research Record, v. 1778, p. 54 - 63. DOI: $10.3141 / 1778-07$

Johnston, D. P.; SurdahL, R. W. (2006). Effects of base type modelling long-term pavement performance of continuously reinforced concrete sections. Transportation Research Record, v. 1979, p. 93 - 101. DOI: $10.3141 / 1979-14$

Kim, S. M.; Won, M. C.; Mccullough, B. F. (2002) Dynamic stresses response of concrete pavements to moving tandem-axle loads. Transportation Research Record, n. 1809, p. 32-41. DOI: 10.3141/1809-04

Kohler, E.; Roesler, J. (2004). Active crack control for continuously reinforced concrete pavements. Transportation Research Record, v. 1900, p. 19 - 29. DOI: 10.3141/1900-03

Liu, C.; Wang Z.; Lee, J. N. (2008) Influence of concrete joints on roughness index and pavement serviceability. International Journal of Pavement Research and Technology, v. 1, n. 4, p. 143-147.

Mccullough, B. F.; Dossey, T. (1999). Considerations for highperformance concrete paving. Recommendations from 20 years field experience in Texas. Transportation Research Record, v. 1684 , p. 17 - 24. DOI: $10.3141 / 1684-03$

Pereira, D. S.; Balbo j. T. (2001) Gradientes térmicos em whitetopping ultradelgado na pista experimental instrumentada da USP. Transportes, v. 9, p. 69-87. DOI:

10.4237/transportes.v18i3.450 
Rodolfo, M. P.; Balbo J. T. (2010) Modelos para

dimensionamento de pavimentos de concreto simples submetidos a carregamentos rodoviários e ambientais empregando análise multivariada de dados. Transportes, v. 18, p. 42-50. DOI: 10.4237/transportes.v9i1.180

Salles, L. S.; Balbo, J. T.; Massola, A. M. A; Pereira, D. S. (2012) Análise de desempenho inicial de um pavimento de concreto continuamente armado de curta extensão. Anais do XXVI Congresso Nacional de Pesquisa e Ensino em Transportes, ANPET, Joinville.

Salles, L. S.; Balbo J. T.; Pereira, D. S. (2013) Crack pattern characterization in a short experimental continuously reinforced concrete pavement. Proceedings of the 2013 International Journal of Pavements Conference, IJPC, São Paulo.

Schindler, A. K.; Mccullough, B. F. (2002). Importance of concrete temperature control during concrete pavement construction in hot weather conditions. Transportation Research Record, v. 1813, p. 3 - 10. DOI: 10.3141/1813-01

Shahin, M. Y. (1985). Use of the falling weight deflectometer for the non-destructive deflection testing of jointed concrete airfield pavements. Proceedings of the 3rd International Conference on Concrete Pavement Design and Rehabilitation, Purdue University, p. 549-556.

Silva, P. D. E. A.; Motta, L. M. G (1999). Instrumentação da pista circular experimental do IPR/DNER. Transportes, v. 7, $\mathrm{p}$ $29-46$

Tayabji, S. D.; Stephanos, P. J; Zollinger, D. G. (1995). Nationwide field investigation of continuously reinforced concrete pavements. Transportation Research Record, v. 1482, p. $7-18$.

Won, M. C. (2011). Continuously reinforced concrete pavement: identification of distress mechanisms and improvement of mechanistic-empirical design procedures. Transportation Research Record, v. 2226, p. 51 - 59. DOI: 10.3141/2226-06

Xiao, T., Sun, J., Wang, X., and Chen, Z. (2011) Dynamic Response Analysis of Cement Concrete Pavement under Different Vehicle Speeds. In: Pavements and Materials: Recent Advances in Design, Testing and Construction (GeoHunan 2011) p. 1-9. DOI: $10.1061 / 47623(402) 1$

Zhang, W.; Wang, G.; Ma, S; Li, X. (2007) Field experimental study on measurement and analysis strain on the rigid pavement slab subjected to moving vehicle loads. Proceedings of the International Conference on Transportation Engineering (ICTE), ASCE, China.

Zollinger, D. G.; Barenberg, E. J. (1990) Mechanistic design considerations for punchout distress in continuously reinforced concrete pavement. Transportation Research Record, v. 1286, p. $25-37$. 